\title{
IoT Soil Monitoring based on LoRa Module for Oil Palm Plantation
}

\author{
Ahmad Alfian Ruslan ${ }^{1}$, Shafina Mohamed Salleh ${ }^{2}$ \\ Sharifah Fatmadiana Wan Muhamad Hatta ${ }^{3}$, Aznida Abu Bakar Sajak ${ }^{4} *$ \\ Computer Engineering, MIIT, Universiti Kuala Lumpur, Kuala Lumpur, Malaysia ${ }^{1,4}$ \\ Computer and Network Engineering, Gerik Vocational College, Kuala Lumpur, Malaysia ${ }^{2}$ \\ Electrical and Electronics Engineering, University of Malaya, Kuala Lumpur, Malaysia ${ }^{3}$
}

\begin{abstract}
Internet of Things (IoT) Soil Monitoring based on Low Range (LoRa) Module for Palm Oil Plantation is a prototype that sends data from the sender to the receiver by using LoRa technology. This realises the implementation of Industrial Revolution 4.0 in the agriculture sector. Also, this prototype uses the TTGO development board for Arduino with built-in ESP32 and LoRa, pH sensor and moisture level sensor as main components. The prototype utilises the LoRa communication between the sender and the receiver. The sensors will detect soil $\mathrm{pH}$ along with the moisture level. The data then will be sent to the receiver, where it will be displayed in the Organic Light-Emitting Diodes (OLED) display. At the same time, the data will be uploaded to the database named ThingSpeak by using wireless communication. Users can monitor the data collected by accessing ThingSpeak's website using smartphones or laptops. The prototype is easy to set up and use to help users monitor the $\mathrm{pH}$ level and moisture level percentage. For future enhancement, the project can be enhanced by combining temperature and tilt sensors to get comprehensive data about the soil's condition.
\end{abstract}

Keywords-Internet of Things (IoT); Low Range (LoRa); Organic Light-Emitting Diodes (OLED); ThingSpeak; Arduino

\section{INTRODUCTION}

This project concerns an Internet of Things (IoT) soil monitoring system using Arduino based on LoRa (Low Range) technology. The project is created for future technology "Industry 4.0" to boost efficiency in palm oil plantation by adopting the IoT in the agriculture sector [1]. Most of the oil palm plantations are in a rural and secluded area where the internet is scarce. Hence, LoRa introduces a new method to realise IoT in the agriculture sector. This is the contribution of this paper. Up to now, palm oil workers manually check and monitor every crop, covering a vast area measuring thousands of hectares [2]. This can be less efficient to monitor each crop for the soil moisture level because oil palm is challenging to grow.

Moreover, after they harvest the crops, they will chop off the old crops to plant new crops. This practice leads to many problems, such as boycotts from climate activists. With the existence of this project, it can reduce the frequency of replanting when monitoring alerts you exactly as soon as it is due for replanting. Oil palm has already been a staple crop, with thousands of hectares being planted and manually monitored by the workers, but it is not the most efficient way to implement it. The workers need to manually check and maintain the appropriate soil moisture level throughout the year. The farmers mostly rely on the little knowledge they gain from experience or by observing crops with naked eyes. This leads to misjudgment, causing devastation to the crops. If a sufficient level of moisture is not maintained, it can be damaging to the crops. For example, maintaining the water supply is crucial to the crops [3-7]. With LoRa and a few sensors, the moisture readings can be viewed easily while also saves time. The design of the monitoring system is utilising the sensor module comprising of temperature sensor and moisture level sensor positioned in the soil that will capture the data taken from the soil. The data then will be transferred to the Arduino development board using the LoRa module.

LoRa is an important part of this project because the plantation lacks internet access and cellular coverage. LoRa was first introduced in the previous work in [8], where the LoRaWAN platform had shown the potential to replace the use of IoT infrastructure on the existing 802.11 Wireless Standard. In [9], an animal movement tracker prototype that used LoRaWAN as a medium to transfer the GPS location of the farmed animals had been built to support smart agriculture. Hence, using LoRa will help to cover the wide radius of the plantation. Unlike the prototype in [10], where the IoT soil monitoring prototype used wireless connectivity as the medium, the LoRa module will act as a network connection to connect to Arduino by using radiofrequency. After that, the data will be kept in the database to be monitored by the plantation workers using mobile devices or laptops.

The scope of this project is to develop an IoT project with the implementation of the LoRa module as the main network connectivity. Using LoRa, the system will have the benefit of low power consumption. Besides, it supports a longer range of coverage area. For measuring data, a $\mathrm{pH}$ sensor is needed to measure acidity or alkalinity with a range from 0 to 14 . Another sensor is a moisture sensor, which is needed to detect moisture level in the soil and manage irrigation systems (the system will turn on when moisture level falls below a certain predefined threshold value) if available. A mobile application will be developed as well to present the data for the workers.

In summary, this project is created to provide a solution to overcome the manual monitoring system used by the workers for a very long time. The IoT monitoring system will ensure to increase productivity and efficiency of the planter at the tip of their finger.

\footnotetext{
*Corresponding Author
} 
This paper will discuss the methodology of the project, the hardware and software development, the results and discussion and the conclusion.

\section{METHODOLOGY}

The research methodology section provides a step by step guideline to complete the project. A suitable methodology for this monitoring system can ensure the success of the project. In this section, the explanation of the chosen methodology as shown in Fig. 1, along with the description of how it is applied in developing a prototype, will be discussed. The result will also be discussed to gather as much information as possible about soil monitoring using the LoRa module. Fig. 2 is the flowchart of the project.

\section{A. Feasibility Study}

A feasibility study is an analysis or evaluation for the project proposed, either it is feasible to be implemented. Thus, the feasibility study consists of the problems statement, objectives, and phases to complete the project. The developer must have various strategies or mitigate planning if something wrong happens in terms of resources required, such as the cost of development and the development time.

\section{B. Requirement Analysis}

In this phase, all of the possible requirements and information regarding the project was collected and documented. The important thing to do in this phase is to identify the project's objectives, requirements, and specifications to proceed to the next phase.

\section{System Design}

In the design phase, the prototype was designed. The design must meet the requirements stated in Table I. It will have TTGO ESP32 with a built-in LoRa module, pH sensor, and moisture sensor on the transmitter side. For the receiver part, it will be designed to have TTGO ESP32 with a built-in LoRa module. ThinkSpeak will act as a database to store the data.

\section{Coding}

In the coding phase, the transmitter sent data; the receiver must receive the data. The transmitter (client) hardware consists of a pH sensor and a moisture sensor; both were fixed to the soil, while both the sensor and LoRa module will be installed to the TTGO ESP32. On the other hand, the receiver side consists of Arduino UNO and installed with the LoRa module to receive data from the transmitter. A database was configured with ThingSpeak to store the data. A mobile application was installed in the receiver to view the data. The TTGO ESP32 was configured by using the Arduino IDE from a laptop. The connectivity enables the data to be sent and received by using the LoRa module.

\section{E. Testing}

The project was tested to identify any defects in the system. Each of the connected sensors was tested whether it is working properly. Any defects found in the system were fixed and tested again to ensure that the defects were fixed. The test was conducted by using natural material (soil) to ensure the correct data was collected. This ensures the objectives are achieved. LoRa module was tested to ensure the connectivity between the transmitter and the receiver. ThinkSpeak was able to store the correct $\mathrm{pH}$ and moisture level readings.

\section{F. Maintenance}

In this phase, a maintenance phase was conducted to correct any errors discovered during the product development phases. This ensures developers always improve implementation and enhance the functionalities of the system.

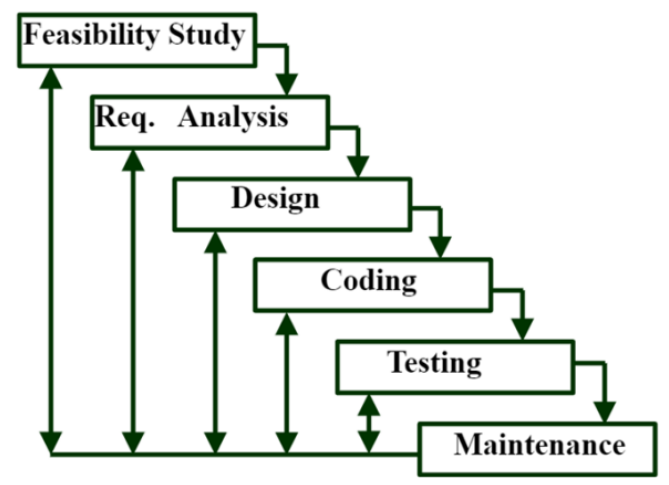

Fig. 1. Iterative Waterfall Model.

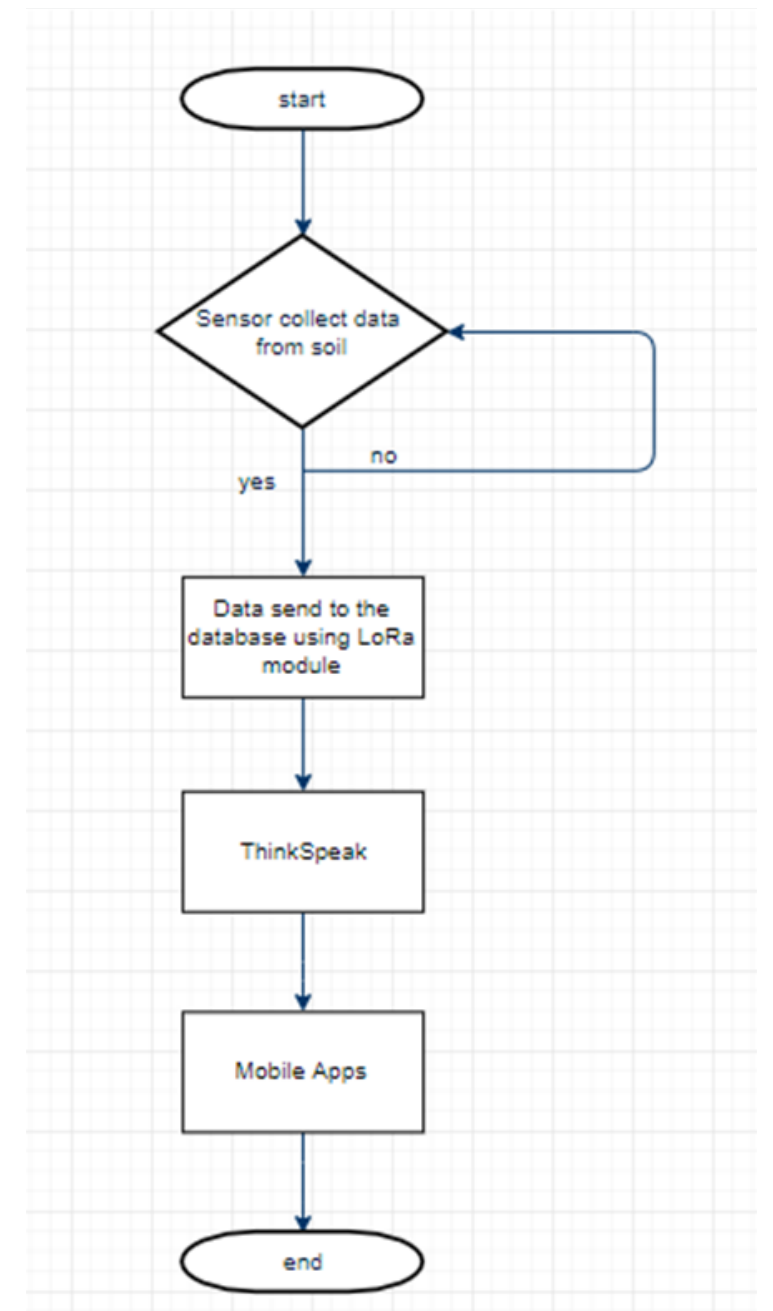

Fig. 2. Project Flowchart. 


\section{HARDWARE AND SOFTWARE}

In this project, the hardware, as shown in Table I, is necessary for development. This step explains the project's purposes, requirements, and specification, including tooling and software. This phase is important for gathering project information to progress to the next phase.

\section{A. TTGO LoRa SX1278 with ESP32}

TTGO LoRa SX1278, shown in Fig. 3, is the main component for both the transmitter and the receiver. This board is built-in together with LoRa SX1278 and ESP32. Thus, the board is working on LoRa and $\mathrm{WiFi}$ communication. The device was used in both the transmitter and the receiver to act as a medium to transmit and receive the data over LoRa communication.

\section{B. Analogue pH Sensor}

Based on Fig. 4, pH sensors are connected to the board TTGO LoRa. There were three pins involved to connect the pH sensor to the TTGO LoRa board.

- $\mathrm{VCC}=+5.5 \mathrm{~V}$ pin

- $\mathrm{GND}=\mathrm{GND}$ pin

- $\mathrm{GPIO}=35$

This sensor can be used to detect the $\mathrm{pH}$ value from soil and water. An example can be seen in Fig. 4. The test was made to test the $\mathrm{pH}$ value from the distilled water.

TABLE I. HARDWARE AND SOFTWARE

\begin{tabular}{|l|l|l|}
\hline \multirow{5}{*}{ Hardware } & Item & Unit \\
\cline { 2 - 3 } & pH sensor & 1 \\
\cline { 2 - 3 } & Moisture sensor & 1 \\
\cline { 2 - 3 } & TTGO LoRa SX1278 ESP32 (OLED) & 1 \\
\cline { 2 - 3 } Software & TTGO LoRa SX1278 ESP32 & 1 \\
\cline { 2 - 3 } & Jumper cable & 1 \\
\hline \multirow{5}{*}{} & Arduino IDE & - \\
\cline { 2 - 3 } & Laptop & - \\
\cline { 2 - 3 } & ThinkSpeak & - \\
\hline
\end{tabular}

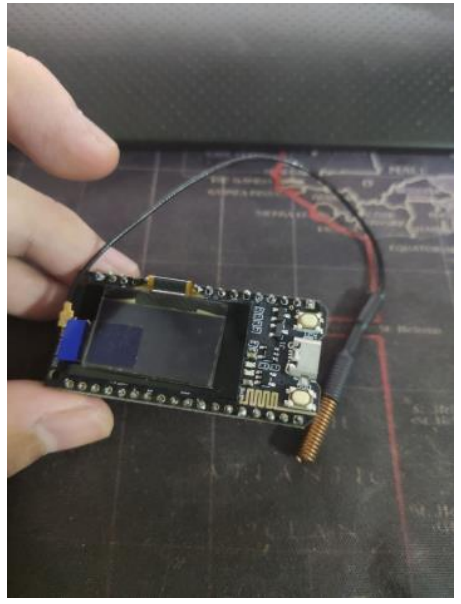

Fig. 3. TTGO ESP32 Build-in LoRa Module.

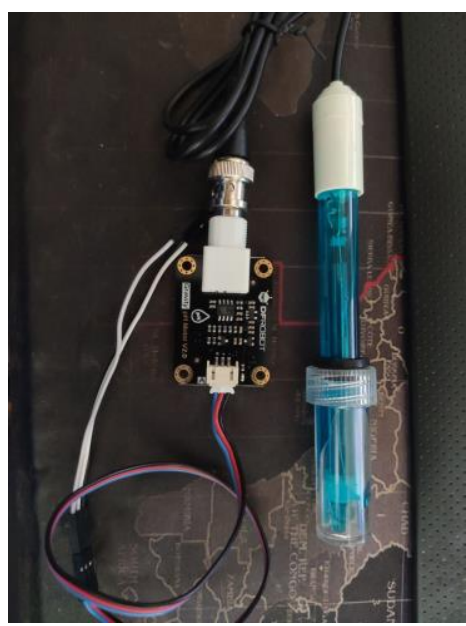

Fig. 4. pH Sensor by DFRobot.

\section{Moisture Sensor}

Based on Fig. 5, this sensor has three pins: VCC, GND, and Analog Output, to connect the sensor to the TTGO board.

- $\quad \mathrm{VCC}=+5.5 \mathrm{~V}$ pin

- $\mathrm{GND}=\mathrm{GND}$ pin

- $\mathrm{GPIO}=32$

This sensor can be used to detect the moisture of water. The sensor will be read in the form of voltage. From the voltage, the higher reading can be interpreted as a high level of moisture. To facilitate reading, the data will be converted into a percentage.

\section{ThingSpeak}

A database is needed to ensure the data received can be collected and processed, and ThingSpeak, as shown in Fig. 6, is the best choice for this. The user accessed the ThingSpeak website to register and create a database channel.

Simple coding, as shown in Fig. 7, was needed at the receiver to ensure the data received was properly sent to the ThingSpeak database. The "apiKey" can be found from the channel. "MY_SSID" \& "MY_PWD" were set up based on the network that connected to the internet.

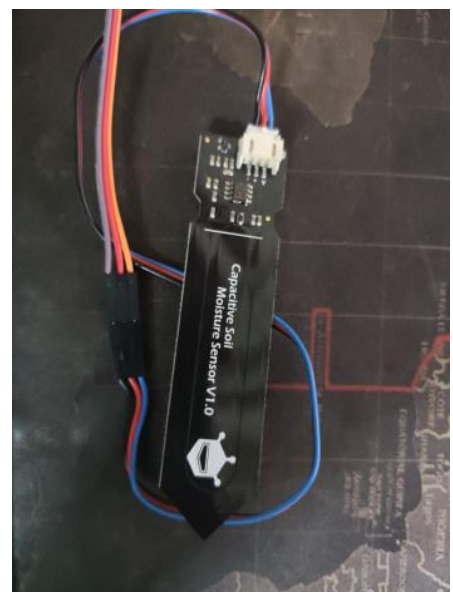

Fig. 5. Moisture Sensor. 


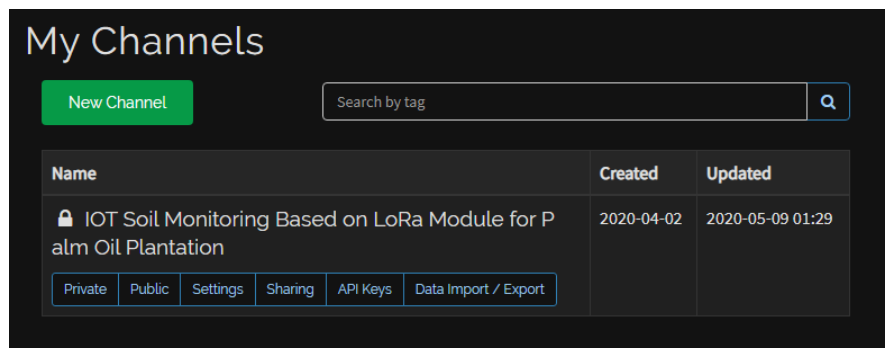

Fig. 6. Project Channel at ThingSpeak.

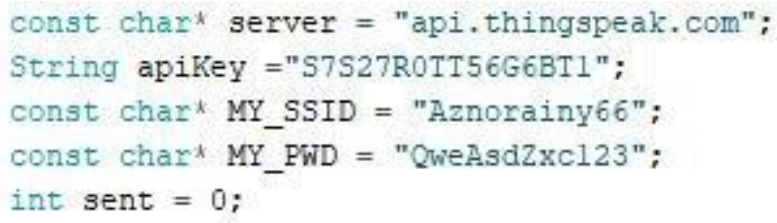

Fig. 7. Code for ThingSpeak.

\section{RESUlT AND DISCUSSION}

Fig. 8 shows the results retrieved from the sensor, stored in the channel's database. In Fig. 8, there were four columns. The first column is to show the exact time when the data was collected. The second was the "entry_id", which was the sequence of data collected. The third was the "field1", the data retrieved from the $\mathrm{pH}$ sensor. The last column was "field2", which was the data from the moisture sensor. All of the data was retrieved together in a packet.

\section{A. Results}

Fig. 9 shows the data from the $\mathrm{pH}$ sensor, which was plotted to a line graph along with the numeric value. For the line graph, the $\mathrm{x}$-axis is the date as per data being collected, while the $\mathrm{y}$-axis is a range of numbers of the $\mathrm{pH}$ readings, which ranged from zero to 14 . The line graph will show us the flow of data collected through the duration setup. The graph was set up to show the latest ten collected data. The numeric value will show the latest data retrieved to make it easier to read.

\begin{tabular}{|c|c|c|c|}
\hline created_at & entry_id & field1 & field2 \\
\hline 2020-06-05 07:08:50 UTC & 483 & 4.83 & 4 \\
\hline 2020-06-05 07:09:20 UTC & 484 & 5.32 & 4 \\
\hline 2020-06-05 07:09:50 UTC & 485 & 5.33 & 5 \\
\hline 2020-06-05 07:10:20 UTC & 486 & 5.33 & 4 \\
\hline 2020-06-05 07:10:50 UTC & 487 & 5.17 & 4 \\
\hline 2020-06-05 07:11:20 UTC & 488 & 5.17 & 4 \\
\hline 2020-06-05 07:11:50 UTC & 489 & 4.91 & 4 \\
\hline 2020-06-05 07:26:12 UTC & 490 & 4.4 & 81 \\
\hline 2020-06-05 07:26:42 UTC & 491 & 4.06 & 81 \\
\hline 2020-06-05 07:27:12 UTC & 492 & 4.88 & 81 \\
\hline 2020-06-05 07:27:42 UTC & 493 & 4.01 & 81 \\
\hline 2020-06-05 07:28:12 UTC & 494 & 4.08 & 81 \\
\hline 2020-06-05 07:28:42 UTC & 495 & 4.07 & 81 \\
\hline 2020-06-05 07:29:12 UTC & 496 & 4.15 & 82 \\
\hline
\end{tabular}

Fig. 8. Database Table.

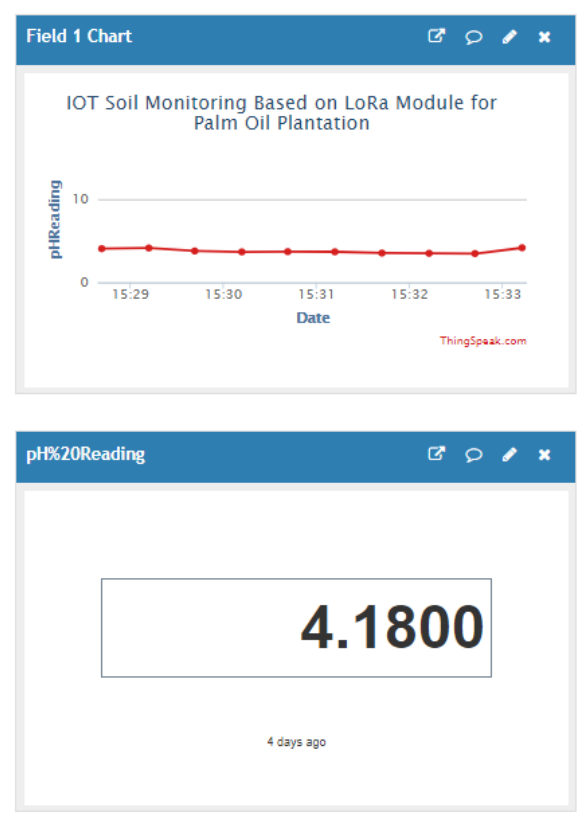

Fig. 9. pH Graph.

In Fig. 10, the data from the moisture sensor was plotted to a line graph, and a numeric value was also displayed. Referring to the line graph, the $\mathrm{x}$-axis is the date as per data being collected, while the y-axis was the range of numbers from zero to 100 , which corresponded to percentages of the moisture level. The graph was set up to show the latest ten collected readings. The numeric value showed the latest data retrieved for ease of reading.

Fig. 11 showed the database could be accessed via smartphones. It has a similar interface as the PC browser interface, which consisted of a line graph and a digital reading of $\mathrm{pH}$ and moisture level. Thus, the users can understand and view the data from their smartphone.

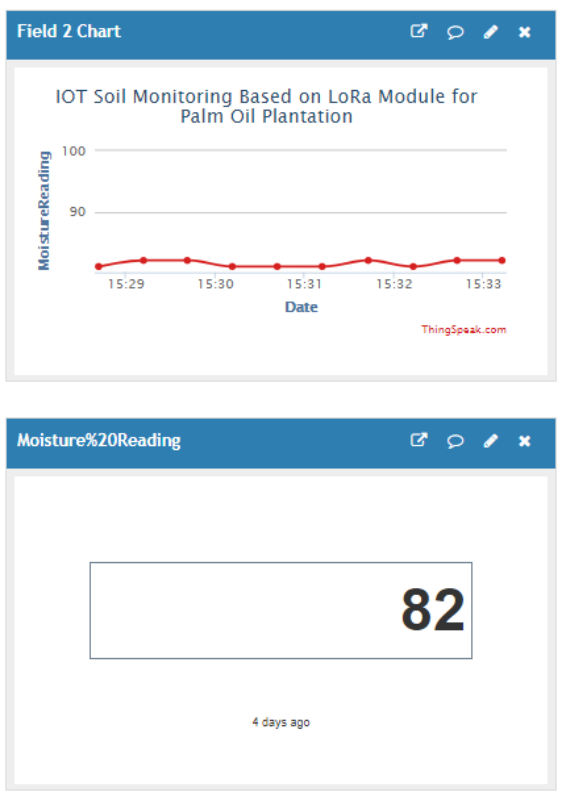

Fig. 10. Moisture Graph. 

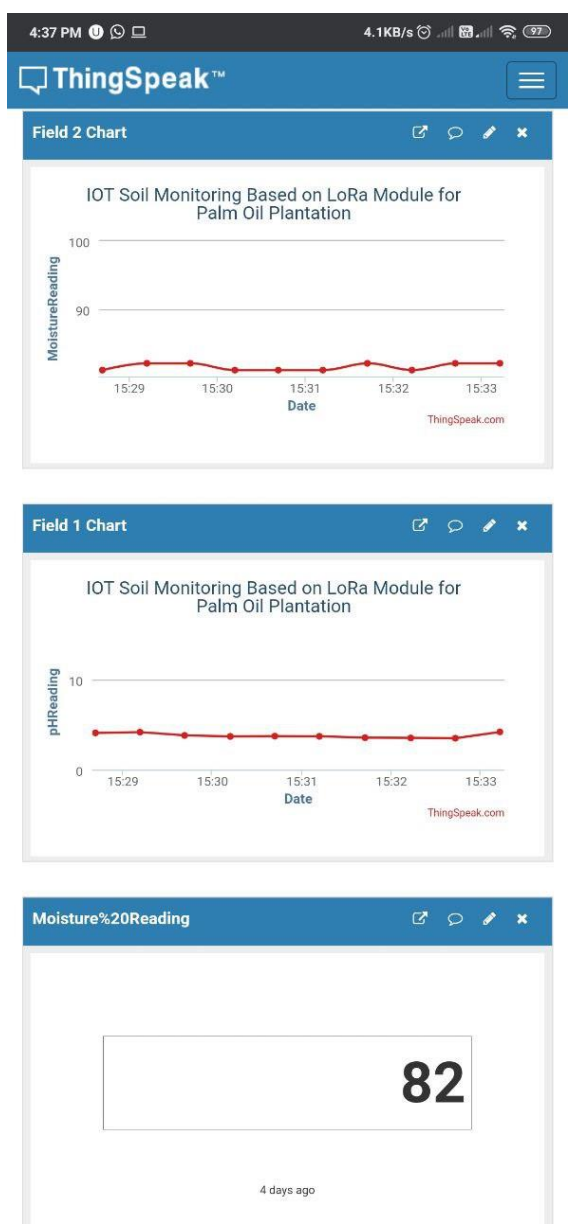

Fig. 11. ThingSpeak via Smartphone view.

\section{B. Analysis}

1) Recommended soil $\mathrm{pH}$ : Fig. 12 shows the soil $\mathrm{pH}$ readings from the ThinkSpeak database in tabular form. The ideal recommended soil $\mathrm{pH}$ for oil palm is between 4.3 to 6.5 [6], which is more acidic. Other readings, aside from the recommended values, will be harmful to the crops. From the table, the entry_id readings between 471 and 481 are ideal. When the readings are between this range, the worker doesn't have to do anything. The worker only takes action if the reading from the sensor is outside the recommended value.

2) Recommended soil moisture: Fig. 13 shows the soil moisture reading from the ThinkSpeak database in tabular form. 80\% [7] soil moisture level is the recommended reading for oil palm. Taking the data from the table as an example, the readings of entry_id between 490 and 494 were sufficient, while the readings between 485 and 489 were insufficient, as this meant that the soil was so dry and can cause the crops to die due to lack of water. Thus, the worker needs to water the plant to moist the soil to the recommended moisture level.

3) LoRa signal analysis: Fig. 14 shows the map of the checkpoints for the testing sites. For this test, there were six different locations to test out the LoRa connectivity. The test sites were located around the residential area and high-rise buildings.

\begin{tabular}{|l|r|r|}
\hline created_at & entry_id & field 1 \\
\hline $2020-06-05$ 07:02:49 UTC & 471 & 5.03 \\
\hline $2020-06-05$ 07:03:19 UTC & 472 & 5.55 \\
\hline $2020-06-05$ 07:03:49 UTC & 473 & 4.92 \\
\hline $2020-06-05$ 07:04:19 UTC & 474 & 5.17 \\
\hline $2020-06-05$ 07:04:49 UTC & 475 & 5.05 \\
\hline $2020-06-05$ 07:05:19 UTC & 476 & 5.25 \\
\hline $2020-06-05$ 07:05:49 UTC & 477 & 5.35 \\
\hline $2020-06-05$ 07:06:19 UTC & 478 & 4.94 \\
\hline $2020-06-05$ 07:06:49 UTC & 479 & 5.09 \\
\hline $2020-06-05$ 07:07:20 UTC & 480 & 5.37 \\
\hline $2020-06-05$ 07:07:50 UTC & 481 & 4.86 \\
\hline
\end{tabular}

Fig. 12. Soil pH Database.

\begin{tabular}{|l|l|l|}
\hline \multicolumn{1}{l|}{ created_at } & entry_id & field2 \\
\hline 2020-06-05 07:09:50 UT & 485 & 5 \\
\hline $2020-06-05$ 07:10:20 UT & 486 & 4 \\
\hline $2020-06-05$ 07:10:50 UT & 487 & 4 \\
\hline $2020-06-05$ 07:11:20 UT & 488 & 4 \\
\hline $2020-06-05$ 07:11:50 UT & 489 & 4 \\
\hline $2020-06-05$ 07:26:12 UT & 490 & 81 \\
\hline 2020-06-05 07:26:42 UT & 491 & 81 \\
\hline $2020-06-05$ 07:27:12 UT & 492 & 81 \\
\hline $2020-06-05$ 07:27:42 UT & 493 & 81 \\
\hline $2020-06-05$ 07:28:12 UT & 494 & 81 \\
\hline
\end{tabular}

Fig. 13. Soil Moisture Database.

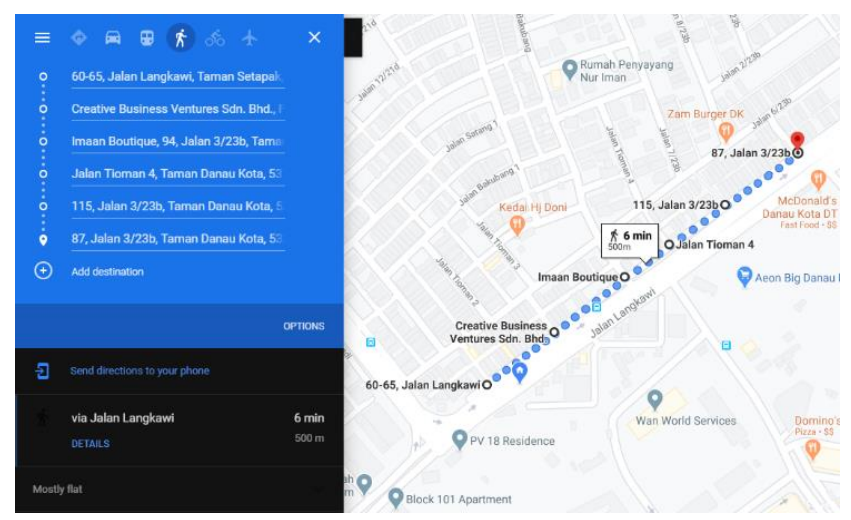

Fig. 14. Checkpoint Test Location.

Table II shows that the distance for each checkpoint, A through G. It showed that maximum LoRa connection was available up to 500 meters, and the connection was lost at checkpoint G. This might be due to interferences coming from cars, motorcycles, trees or might be due to the loss of Line of Sight (LOS). Next, for the received signal strength indicator (RSSI) measurements, the signal was much better as the signal value neared $0 \mathrm{dBm}$. At checkpoint A, the RSSI reading is -79 $\mathrm{dBm}$, while at another checkpoint, RSSI's was greater than $100 \mathrm{dBm}$. When the signal is $-100 \mathrm{dBm}$, and below, the signal strength is not good as the better signal strength readings should be closer to $0 \mathrm{dBm}$.

TABLE II. LORA CONNECTIVITY RESULT

\begin{tabular}{|l|l|l|l|l|l|l|l|}
\hline & A & B & C & D & E & F & G \\
\hline Distance (m) & 5 & 100 & 200 & 300 & 400 & 500 & 700 \\
\hline Connectivity & $\checkmark$ & $\checkmark$ & $\checkmark$ & $\checkmark$ & $\checkmark$ & $\checkmark$ & x \\
\hline RSSI & -79 & -114 & -117 & -116 & -110 & -116 & $\mathrm{x}$ \\
\hline
\end{tabular}




\section{CONCLUSION}

Palm oil production is vital for the Malaysian economy, which is the world's second-largest producer of the commodity, after Indonesia. Thus, the implementation of IoT in this agriculture sector will greatly increase the profit to the country. As internet connectivity is scarce in rural area, where most agriculture sectors are located, Lora had been identified as the solution for data transmission. Using this project, we can monitor every aspect of oil palm management and plantation. With the increasing application of IoT in the sector, the efficiency and productivity will be increased and the profit margin of the palm oil industry. Workers can view and monitor real-time soil condition along with various other agriculture variables via apps or websites. This is because of the sensors installed in the plantation; with the telemetry, all of the data can be displayed on the screen ready to be analysed, and solution can be searched for should there be any problem with the data obtained. Also, unnecessary and troublesome travels across the plantation to monitor the condition of the crop can be minimised. Adding more sensors such as tilt and temperature sensors such as in [11] will result in a more comprehensive prototype that can test the soil's condition more accurately.

\section{REFERENCES}

[1] "Ruler: Industry 4.0 tech lagging in palm oil sector | The Star." [Online]. Available:https://www.thestar.com.my/business/business-news/2019/07/ 16/ruler-industry-40-tech-lagging-in-palm-oil-sector. [Accessed: 09-Jun2020].

[2] "Industrial-scale pyrolysis the easiest to use: The Role of Biochar In Era of Precision Farming and IoT in Palm Oil Plantations." [Online]. Available: http://jfe-project.blogspot.com/2017/07/the-role-of-biocharin-era-of-precision.html. [Accessed: 09-Jun-2020].
[3] R. Dagar, S. Som, and S. K. Khatri, "Smart Farming - IoT in Agriculture," Proc. Int. Conf. Inven. Res. Comput. Appl. ICIRCA 2018, no. Icirca, pp. 1052-1056, 2018.

[4] S. Verma, R. Gala, S. Madhavan, S. Burkule, S. Chauhan, and C. Prakash, "An Internet of Things (IoT) Architecture for Smart Agriculture," Proc. - 2018 4th Int. Conf. Comput. Commun. Control Autom. ICCUBEA 2018, pp. 1-4, 2018.

[5] S. Heble, A. Kumar, K. V. V. D. Prasad, S. Samirana, P. Rajalakshmi, and U. B. Desai, "A low power IoT network for smart agriculture," IEEE World Forum Internet Things, WF-IoT 2018 - Proc., vol. 2018Janua, pp. 609-614, 2018.

[6] R. Rozieta, A. R. Sahibin, and I. Wan Mohd Razi, "Physico-chemical properties of soil at oil palm plantation area, Labu, Negeri Sembilan," in AIP Conference Proceedings, 2015, vol. 1678.

[7] "Plantations International Palm Oil." [Online]. Available: https://www.plantationsinternational.com/palm-oil/. [Accessed: 09-Jun2020].

[8] J. Jaffar, A. F. Abdul Malik, M. Farez Azril Zuhairi, A. A. Bakar Sajak and M. Taha Ismail, "Development of the LoRaWAN-based Movement Tracking System," 2020 14th International Conference on Ubiquitous Information Management and Communication (IMCOM), Taichung, Taiwan, 2020, pp. 1-6, doi: 10.1109/IMCOM48794.2020.9001689.

[9] S. Alimin Mahama Chedaod, A. Abu Bakar Sajak, J.Jaffar, and M. Sallehin Mohd Kassim, "LoRaWAN based Movement Tracker for Smart Agriculture", International Journal of Advanced Trends in Computer Science and Engineering (IJATCSE), Vol.9, No. 1.5, July 2020 , p. $253-258$.

[10] M. Shakhil Iman Hasnan, R. Rawi and A. Abu Bakar Sajak , "Palm Oil Soil Monitoring System for Smart Agriculture", The International Journal of Integrated Engineering, Vol. 12, No. 6, September 2020, p. $189-199$.

[11] M.Syarafuddin Md Saleh, M. Ariff Majmi Zaaba, R. Mohamad and A. Abu Bakar Sajak “ IoT Real-Time Soil Monitoring based on LoRa for Palm Oil Plantation ”, unpublished. 